ORIGINAL ARTICLE

\title{
Epidemiology, clinical characteristics, and management of adults referred to a teaching hospital first seizure clinic
}

\author{
D P Breen, M J G Dunn, R J Davenport, A J Gray
}

Postgrad Med J 2005;81:715-718. doi: 10.1136/pgmj.2004.031203

See end of article for authors' affiliations .....................

Correspondence to: Dr D P Breen, Department of General Surgery, Borders General Hospital, Melrose TD6 9BS,

Scotland; davebreen@ lycos.com

Submitted

1 December 2004

Accepted

23 February 2005
Background: There are scarce data describing the epidemiology, clinical characteristics, and management of adults who suffer a suspected first seizure.

Aim: To describe the epidemiology, clinical characteristics, and management of adults with a suspected first seizure who are referred to a teaching hospital first seizure clinic over a one year period.

Design: Prospective descriptive study.

Methods: Data were collected on consecutive adults referred to the Royal Infirmary of Edinburgh between 4 February 2003 and 10 February 2004.

Results: 232 patients were referred to the first seizure clinic. Median age was 32 years; $53 \%$ of patients were male. Lower socioeconomic groups were more likely to present with a suspected first seizure. Nineteen per cent of patients were admitted to hospital after their suspected seizure episode. Appropriate driving advice was reported in $64 \%$ of cases. Seventy two per cent of patients were offered a first seizure clinic appointment within six weeks of referral. Nine per cent of patients had a subsequent seizure while awaiting review. Fifty two per cent of patients were confirmed as having a first seizure at the clinic, of which $56 \%$ were provoked by alcohol, recreational drugs, or sleep deprivation. Electroencephalography and computed tomography of the brain were the most common investigations ordered at the first seizure clinic ( $22 \%$ and $22 \%$ of patients respectively).

Conclusion: Adults who suffer a suspected first seizure, and who make a full neurological recovery, can be safely managed as an outpatient. Around half of these patients will have a specialist diagnosis of first seizure and alcohol will be a common precipitating factor.
A bout $5 \%$ of the population will experience a non-febrile seizure during their lifetime. ${ }^{12}$ One third of these patients will have a further unprovoked seizure, at which time they will be diagnosed with epilepsy. ${ }^{3}$ Most first seizure patients present to an emergency department (ED). ${ }^{4} 5$ In Edinburgh, a clinical pathway has been developed for adults who present to the ED with a suspected first generalised seizure. ${ }^{6}$ Patients also present to their general practitioner (GP) after a suspected first seizure episode.

It is recommended that all adults who suffer a suspected first seizure should be seen by an epilepsy specialist in a dedicated first seizure clinic. ${ }^{7}$ There are scare data describing the epidemiology of these patients, and there continues to be lack of clarity as to how they should be investigated and managed.

The aim of this study was to describe the epidemiology, clinical characteristics, and management of adults with a suspected first seizure who are referred to a teaching hospital first seizure clinic over a one year period.

\section{METHODS}

Data were collected on consecutive adults referred to the first seizure clinic at the Royal Infirmary of Edinburgh between 4 February 2003 and 10 February 2004. These patients were prospectively identified. Hospital records were obtained and the following information was recorded:

\section{Patient characteristics}

Sex, age, postcode, primary route of referral, and date of referral were identified from the patient's notes. Socioeconomic status was measured using Carstairs deprivation categories. ${ }^{8}$ These are based on residential postcodes and other indicators of socioeconomic status.

\section{Management in ED lonly patients referred via ED} included in this section)

Notes were scrutinised to identify day and time of presentation, ED observations, electrocardiogram (ECG) findings, blood test results, and ED management decisions. Injuries suffered as a result of the seizure, inpatient medical referrals, and any documentation of driving advice was also recorded.

\section{First seizure clinic}

The date of clinic appointment, specialist diagnosis including seizure type, possible precipitants of seizure, and additional investigations ordered were recorded.

\section{Ethical approval and statistical analysis}

The study was approved by Lothian local research ethics committee. Non-continuous variables are described as number of patients (along with percentages). The 95\% confidence intervals are given for the observed differences between variables.

\section{RESULTS}

Altogether, 296 patients were referred to the first seizure clinic between 4 February 2003 and 10 February 2004. Hospital records were obtained for 286 patients (97\%). We excluded 37 patients with non seizure-related symptoms who had been booked into the clinic in error, and 17 patients who had a pre-existing diagnosis of epilepsy. Therefore, 232 patients were included in the final analysis.

The mean age of presentation was 37 years (median 32; range 13-84); 123 patients (53\%) were male. First seizure clinic patients were significantly less likely to be from Carstairs deprivation categories 3 and 4, and more likely to be from deprivation category 5, compared with the Lothian population (fig 1). A total of 124 patients (53\%) were referred 


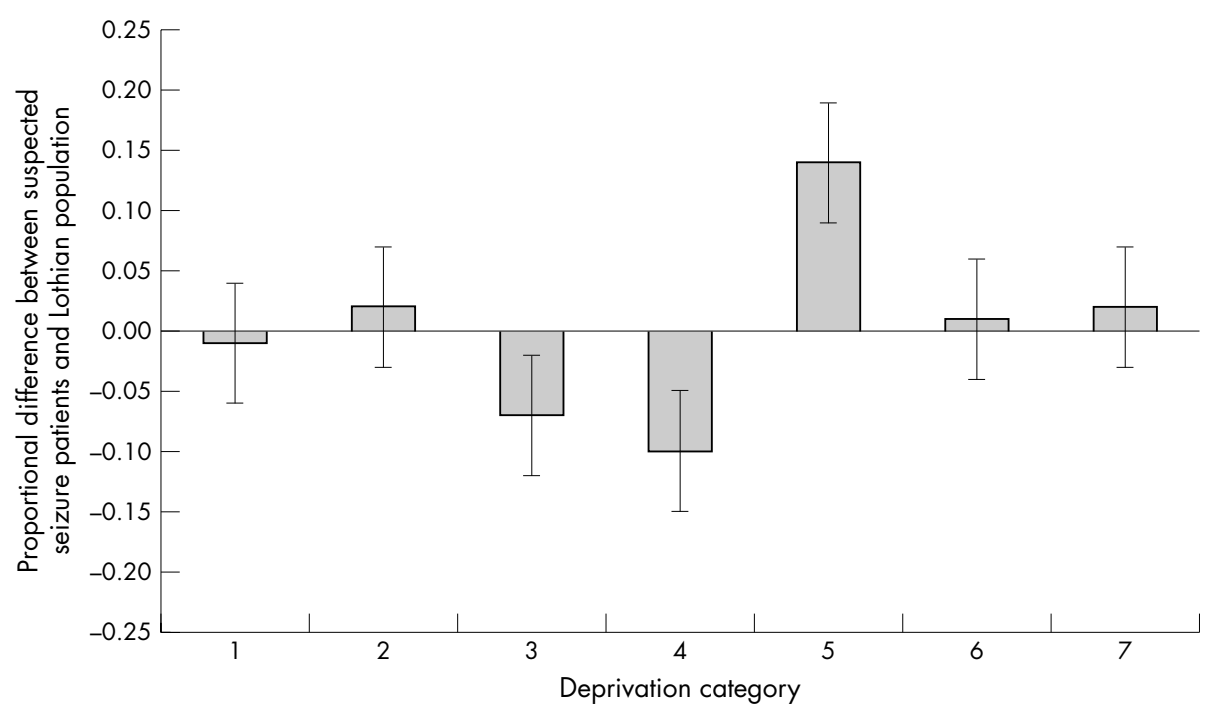

Figure 1 Comparison between socioeconomic status of suspected seizure patients $(n=232)$ and Lothian population $(n=756165)$. Carstairs deprivation categories run from category 1 (most affluent) to category 7 (most deprived). This graph shows the proportional difference between suspected seizure patients and the Lothian population, along with the $95 \%$ confidence intervals. First seizure clinic patients were significantly less likely to be from deprivation categories 3 and 4 , and more likely to be from deprivation category 5, compared with the Lothian population.

to the first seizure clinic from the ED, 78 patients (34\%) by their GP, and 30 patients (13\%) from hospital wards.

\section{ED management}

Most patients who presented to the ED had a normal temperature, pulse rate, and blood pressure. Three patients (3\%) were febrile (temperature $>37^{\circ} \mathrm{C}$ ), 25 patients $(17 \%)$ were tachycardic (pulse $>100 \mathrm{bpm}$ ), and 27 patients $(18 \%)$ were hypertensive (systolic blood pressure $>140 \mathrm{~mm} \mathrm{Hg}$ ). Breath alcohol concentration (BAC) was measured in 39 patients $(25 \%)$. BAC was detectable in 14 patients (range 10 $80 \mathrm{mg} \%$ ). Glasgow coma score (GCS) was 15 in all patients on discharge from the ED.

A total of 136 patients (88\%) had an ECG in the ED. Six patients $(4 \%)$ had a recorded abnormality (two $\mathrm{T}$ wave inversion, two right bundle branch block, one left ventricular hypertrophy, and one first degree heart block). Sixty six patients $(43 \%)$ were hyperglycaemic, 47 patients $(31 \%)$ had a raised white cell count, 29 patients (19\%) had increased $\gamma$ glutamyl transferase activity, and 26 patients $(17 \%)$ had a low total venous carbon dioxide. No single blood test was associated with a final diagnosis of first seizure when the results were statistically analysed.

Before being seen at the first seizure clinic, 26 patients (17\%) had computed tomography (CT). The scan was normal in 20 patients $(77 \%)$, abnormal in one patient (4\%) (evidence of previous stroke), and the report was not available in five patients $(19 \%)$. Seven patients $(5 \%)$ underwent electroencephalography (EEG) before the follow up clinic. This showed normal activity in four patients (57\%), focal epileptic activity in two patients (29\%), and the report was not available in one patient (14\%).

Three patients $(2 \%)$ were prescribed anticonvulsant drugs in the ED. Four patients (3\%) suffered an injury as a result of their suspected seizure that required ongoing management (one orbital fracture, one vertebral fracture, one nasal fracture, and one dental damage). Twenty nine patients (19\%) were admitted to hospital after their suspected seizure episode. Thirty four of the 53 drivers $(64 \%)$ had recorded evidence that Driver and Vehicle Licensing Agency (DVLA) advice had been given in the ED.

\section{First seizure clinic}

A total of 168 patients (72\%) were offered an appointment at the first seizure clinic within six weeks of referral. Of the remaining 64 patients $(28 \%)$, there was an obvious reason for
Table 1 Possible precipitants of seizure

\begin{tabular}{ll}
\hline Precipitant & Number (\%) \\
\hline Alcohol excess & $25(26)$ \\
Sleep deprivation & $16(16)$ \\
Head injury & $11(11)$ \\
Drug misuse* & $9(9)$ \\
Alcohol withdrawal & $5(5)$ \\
Prescription drugt & $3(3)$
\end{tabular}

*Misused drugs: benzodiazepines ( $n=3)$, cannabis $(n=3)$, cocaine $(n=2)$, and amphetamines $(n=1)$. †Prescription drugs: clomipramine $(n=1)$, venlafazine $(n=1)$, and trazodone $(n=1)$.

the delay in $25 \%$ of cases ( 10 did not attend their original appointment, three were not referred straight away, and two referrals were not received). Unfortunately, 42 patients (18\%) did not attend their clinic appointment. Data on the remaining 190 patients were therefore available for analysis.

Eighteen patients (9\%) suffered a further suspected seizure while waiting to be seen at the first seizure clinic. Ninety eight patients $(52 \%)$ were diagnosed as having a seizure. Other specialist diagnoses included syncope in 54 patients $(28 \%)$, non-epileptic episode in four patients $(2 \%)$, hyperventilation in two patients ( $1 \%)$, panic attack in two patients $(1 \%)$, and cardiac arrhythmia in one patient $(1 \%)$. Twenty nine patients ( $15 \%$ ) had no cause identified for their collapse.

Of the patients diagnosed as having a first seizure, 9l patients $(93 \%)$ suffered a generalised tonic-clonic seizure, four patients $(4 \%)$ suffered a partial seizure, and three patients $(3 \%)$ suffered a myoclonic seizure. Table 1 shows the possible precipitants in those patients with a diagnosed first seizure.

Excessive alcohol consumption was the most common possible precipitating factor. Fifteen patients (15\%) were thought to have suffered more than one generalised seizure during their life, although these had not been confirmed by a doctor.

The most commonly ordered investigations at the first seizure clinic were EEG in 42 patients $(22 \%)$ and CT in 42 patients $(22 \%)$. CT scan was normal in 26 patients $(62 \%)$, abnormal in eight patients (19\%) (four previous strokes, two significant atrophy, one bifrontal gliosis and evidence of surgery, and one arteriovenous malformation), and the report was not available in eight patients (19\%). EEG showed normal brain activity in 17 patients $(40 \%)$, focal epileptic activity in 10 patients (24\%), was equivocal in five patients 
$(12 \%)$, and the report was not available in 10 patients $(24 \%)$. MRI was requested in 16 patients (8\%). The scan was normal in nine patients $(56 \%)$, abnormal in five patients $(31 \%)$ (one carcinoma, one arachnoid cyst, one vasculitis, one previous stroke, and one arteriovenous malformation), and the report was not available in two patients (13\%). A 12-lead ECG was requested in 24 patients (13\%), 24 hour ECG in six patients $(3 \%)$, echocardiography in one patient $(1 \%)$, and hyperventilation studies in one patient $(1 \%)$.

\section{DISCUSSION}

This study describes the epidemiology, clinical characteristics, and management of adults with a suspected first seizure who were referred to a teaching hospital first seizure clinic over a one year period.

Age, sex, day and time of presentation, and hospital admission rate were not significantly different from all ED attendances at The Royal Infirmary of Edinburgh between 1 August 2003 and 31 July 2004. However, first seizure clinic patients were more likely to be from lower socioeconomic groups than the Lothian population. This has been reported previously for other acute medical presentations. ${ }^{10}$ It can only be hypothesised whether this is attributable to an increased incidence of medical problems in this patient group or an increase in healthcare seeking behaviour.

A clinical pathway for adults with a suspected first seizure has been designed and implemented in Edinburgh on the basis that most first seizure patients present to the ED. One third of patients in our study were referred to the first seizure clinic by their GP. This is higher than expected and suggests that the pathway should be more widely implemented so that basic observations and investigations can be carried out before first seizure clinic review.

Evidence suggests that the incidence of new onset seizures in the ED attributable to correctable metabolic abnormalities is low. ${ }^{11}{ }^{12}$ Indeed, no single blood test was associated with a final diagnosis of first seizure in our population. However, routine blood tests are important as any abnormality may influence management and the need for admission. On admission to the ED, $31 \%$ of patients with a diagnosed first seizure had an increased white cell count, which probably reflects adrenergic mediated leucocyte demargination. ${ }^{11}$ Forty three per cent of patients were hyperglycaemic, which may suggest an acute stress response, ${ }^{13}$ although serum glucose may have been increased before the seizure. Nineteen per cent of patients had a raised $\gamma$-glutamyl transferase activity, which may reflect chronic alcohol consumption in the patient population studied. Seventeen per cent of patients had a low total venous carbon dioxide, which is indicative of metabolic acidosis.

Adults with a suspected first seizure can be safely managed as an outpatient providing they have made a full neurological recovery. Only those with persistent neurological symptoms or signs, or abnormal observations or investigations, need to be admitted to hospital. In Edinburgh, 19\% of patients were admitted to hospital. Previous studies have reported much higher admission rates. ${ }^{4}{ }^{14}$ Surprisingly, only 3\% of patients suffered a significant injury as a result of their suspected seizure.

The first seizure clinic is the most appropriate setting in which to order specialist investigations, unless they are urgently indicated in the ED. Seventeen per cent of patients had a CT scan before being seen at the first seizure clinic, while 5\% underwent an EEG before the clinic. Two per cent of patients were prescribed anticonvulsant drugs in the ED, presumably because the risk of seizure recurrence was thought to be particularly high. ${ }^{7}$ It is our opinion that prophylactic antiepileptic drugs should not routinely be started in the ED or primary care, but only after consultation with a neurologist or other specialist with an interest in epilepsy.

The proportion of patients in whom driving advice was reorded was much higher than has been reported elsewhere ${ }^{41516}$ but was still suboptimal. All patients with a suspected first generalised seizure should be advised to stop driving when they are initially seen, and it should be recorded in the notes that this counselling has taken place. ${ }^{4}$ In Lothian, a first seizure information leaflet has been used for this purpose. ${ }^{17}$ The patients should subsequently notify the DVLA if the diagnosis is confirmed at the follow up clinic. ${ }^{18}$

All patients who suffer a suspected first seizure should be seen be an epilepsy specialist as quickly as possible. The Scottish Intercollegiate Guidelines Network (SIGN) guidelines state that "the diagnosis of epilepsy is most appropriately delivered in the setting of a dedicated first seizure clinic ${ }^{\prime \prime} .{ }^{7}$ Currently, $72 \%$ of suspected first seizure patients are offered an appointment at the Edinburgh first seizure clinic within six weeks of referral. Nine per cent of patients had a further suspected seizure while waiting to be seen at the follow up clinic.

A first seizure was diagnosed in $52 \%$ of cases at the follow up clinic. This type of over-referral is inevitable but completely acceptable given the difficulty in distinguishing seizures from other mimics. Such referrals are important because they avoid unnecessary investigations and treatments. Fifty six per cent of seizures were thought to have been provoked by either alcohol, recreational drugs, or sleep deprivation. This is higher than has been reported previously. ${ }^{45}$ This is significant because such seizures are avoidable if the patient can avoid such precipitants.

EEG and CT were the most common investigations ordered in the first seizure clinic ( $22 \%$ and $22 \%$ of cases respectively). This is interesting given that the SIGN guidelines state that "MRI is the modality of choice for brain imaging in patients with epilepsy". ${ }^{7}$ The results of this study have led to a change in referral patterns for brain imaging in Edinburgh, and MRI is now becoming the imaging modality of choice for first seizure patients. This clearly has implications for service provision. It is important to note that the abnormalities found on neuroimaging may not necessarily have been responsible for the patient's seizure. In addition, the comparatively high number of patients with abnormalities on neuroimaging and epileptic activity on EEG is probably attributable to the highly selected nature of this group of patients. Namely, that these patients were felt to have suffered a secondary generalised tonic-clonic seizure and so were more likely to have a focal epileptic source.

This is the first study to describe both the early management and follow up of first seizure patients. One of the strengths of the study is that multiple sources were used to collect relevant patient data. These included hospital records, ED records, and laboratory blood results. All patients who presented to the ED were managed according to an established algorithm ${ }^{6}$ and all patients were seen by the same consultant neurologist at the follow up clinic. Inevitably, there will be some patients who were not appropriately referred to the first seizure clinic, or who were referred to another outpatient clinic. These patients could not be identified. We also used Carstairs deprivation categories as an estimate of socioeconomic status. These deprivation categories were published in 1991 and hence may be outdated. Having said this, they are still widely used as a public health research tool instead of newer methods such as the Scottish index of multiple deprivation. ${ }^{19}$

\section{CONCLUSION}

Adults who suffer a suspected first seizure, and who make a full neurological recovery, can be safely managed as an 
outpatient. Around half of these patients will have a specialist diagnosis of first seizure and alcohol will be a common precipitating factor.

\section{ACKNOWLEDGEMENTS}

The authors thank Nicky Kane and Cath Stevenson for their help with data collection. Thanks also to Rik Smith and Jenny Henry for their help with the statistical analysis, Jillian Campbell for providing the sociodeconomic data, Dave Sorenson for providing the ED attendance data, and Fiona McKinnon for producing the first seizure information leaflet. We are also grateful to the neuroimaging and EEG staff at The Royal Infirmary of Edinburgh at Little France and the Western General Hospital for their assistance.

\section{CONTRIBUTORS}

David Breen collected and analysed the data, and wrote the paper Mark Dunn wrote the paper. Richard Davenport prospectively identified all patients referred to the first seizure clinic and edited the paper. Alasdair Gray edited the paper and will act as guarantor for the paper

\section{Authors' affiliations}

D P Breen, Department of General Surgery, Borders General Hospital, Melrose, Scotland

M J G Dunn, A J Gray, Emergency Department, The Royal Infirmary of Edinburgh at Little France, Edinburgh, Scotland

R J Davenport, Department of Clinical Neurosciences, Western General Hospital, Edinburgh, Scotland

Funding: the Department of Clinical Neurosciences received a grant from GlaxoSmithKline to fund the first seizure clinic in its first year.

Competing interests: none declared.

\section{REFERENCES}

1 Forsgren L, Bucht G, Eriksson S, et al. Incidence and clinical characterization of unprovoked seizures in adults: a prospective population-based study. Epilepsia 1996;37:224-9.
2 Hauser WA, Annegers JF, Kurland LT. Incidence of epilepsy and unprovoked seizures in Rochester, Minnesota; 1935-1984. Epilepsia 1993;34:453-68

3 Berg AT, Shinnar S. The risk of seizure recurrence following a first unprovoked seizure. Neurology 1991;41:965-72.

4 Morrison AD, McAlpine $\mathrm{CH}$. The management of first seizures in adults in a district general hospital. Scott Med J 1997;42:73-5.

5 King M, Newton M, Jackson G, et al. Epileptology of the first-seizure presentation: a clinical, electroencephalographic, and magnetic resonance imaging study of 300 consecutive patients. Lancet 1998;352:1007-11.

6 Dunn MJG, Breen DP, Davenport RJ, et al. Early management of adults with an uncomplicated first generalised seizure. Emerg Med J 2005:22:237-42.

7 Scottish Intercollegiate Guidelines Network. Diagnosis and management of epilepsy in adults. Edinburgh: SIGN, 2003.

8 Carstairs V, Morris R. Deprivation and health in Scotland. Aberdeen: Aberdeen Press, 1991

9 Morgan J, Cullen W, Bury G, et al. Profile of attendance at a maternity hospital emergency room. Ir J Med Sci 2000;169:122-4.

10 Round A. Emergency medical admissions to hospital-the influence of supply factors. Public Health 1997;111:221-4.

11 Turnbull TL, Vanden Hoek TL, Howes DS. Utility of laboratory studies in the emergency department patient with a new-onset seizure. Ann Emerg Med 1990; 19:373-7.

12 Powers RD. Serum chemistry abnormalities in adult patients with seizures. Ann Emerg Med 1985; 14:416-20.

13 Capes SE, Hunt D, Malmberg K, et al. Stress hyperglycaemia and prognosis of stroke in nondiabetic and diabetic patients: a systematic overview. Stroke $2001 ; 32: 2426-32$.

14 Henneman PL, DeRoos F, Lewis RJ. Determining the need for admission in patients with new-onset seizures. Ann Emerg Med 1994;24:1108-14.

15 Edmundstone WM. How do we manage the first seizure in adults? J R Coll Physicians 1995;29:289-94.

16 Ryan J, Nash S, Lyndon J. Epilepsy in the accident and emergency department-developing a code of safe practice for adult patients. South East and South West Thames Accident and Emergency Specialty Sub-committees. $J$ Accid Emerg Med 1998;15:237-43.

17 NHS Lothian. First seizure information leaflet. Edinburgh: NHS Lothian, 2003.

18 Driving and Vehicle Licensing Agency. DVLA-at a glance. Neurological disorders. http://www.dvla.gov.uk/at_a glance/chl neurological laccessed 10 Sep 2004).

19 Department of Social Policy and Social Work. Scottish indices of multiple deprivation. Oxford: University of Oxford, 2003, http:// www.scotland.gov.uk/library5/social/siod-00.asp (accessed 10 Sep 2004). 